\title{
Factores pronósticos en adultos hospitalizados por neumonía causada por Legionella pneumophila
}

\author{
María Virtudes Regueiro-Mira, Salvador Pita-Fernández, Sonia Pértega-Díaz, Beatriz López-Calviño, \\ Teresa Seoane-Pillado y María Fernández-Albalat-Ruiz
}

\section{Prognostic factors in adult patients hospitalized for pneumonia caused by Legionella pneumophila}

Introduction: Legionellosis is a multisystem bacterial disease, which causes pneumonia with high mortality in patients with comorbidity and admitted in intensive care units (ICU). Objetive: Determine predictors of mortality or ICU admission. Methods: Retrospective follow-up of patients diagnosed with Legionella pneumophila pneumonia in Complexo Hospitalario Universitario de A Coruña. Period 2000-2013 ( $\mathrm{n}=240)$. Analysis of multivariate logistic regression was performed. Results: Mean age was $57.2 \pm 15.4$ years old, $88.3 \%$ were male. Average score of comorbidity (Charlson score) was $2.3 \pm 2.3$. There was a clear seasonal variation. Predominant symptoms were fever (92.5\%), dry cough (38.1\%) and dyspnea (33.9\%). Creatinine clearance was lower than $60 \mathrm{~mL} / \mathrm{min} / 1.73$ $\mathrm{m}^{2}$ in $29.7 \%$ and sodium $<135 \mathrm{mEq} / 1$ in $58.3 \%$. Admission to ICU rate was $16.3 \%$ and $10.8 \%$ needs mechanical ventilation. Inhospital mortality rate was $4.6 \%$, rising to $23.1 \%$ in patients admitted to ICU. Variables associated to predict ICU admission were age $(\mathrm{OR}=0.96)$, liver disease $(\mathrm{OR}=7.13)$, dyspnea $(\mathrm{OR}=4.33)$, delirium $(\mathrm{OR}=$ $5.86)$ and high levels of lactatedehydrogenase $(\mathrm{OR}=1.002)$. Variables associated with inhospital mortality were Charlson index $(\mathrm{OR}=1.70)$, mechanical ventilation $(\mathrm{OR}=31.44)$ and high levels of lactatedehydrogenase $(\mathrm{OR}$ =1.002). Discussion: Younger patients with liver disease, dyspnea and confusion are more likely to be admitted to ICU. Comorbidity, mechanical ventilation and elevated LDH levels are associated with higher mortality rate.

Key words: Legionella pneumophila; Intensive Care Unit; inhospital mortality; comorbidity; lactate dehydrogenase.

Palabras clave: Legionella pneumophila; Unidad de Cuidados Intensivos; mortalidad intrahospitalaria; comorbilidad; lactato deshidrogenasa.

\section{Introducción}

\section{$\mathrm{L}$}

a legionelosis es una enfermedad bacteriana aguda multisistémica, causada, en más de $85 \%$ de los casos, por el bacilo gramnegativo Legionella pneumophila serogrupo $1^{1-3}$, con manifestaciones clínicas y epidemiológicas diferentes: la enfermedad del legionario y la fiebre de Pontiac. Generalmente aparece como caso aislado, pero puede presentarse como epidemias. Legionella pneumophila es una especie ubicua, acuática, que crece de forma óptima con una temperatura ambiental de 35 a $37^{\circ} \mathrm{C}$. El origen más frecuente es la eliminación de aerosoles producidos por torres de refrigeración e instalaciones de agua caliente ${ }^{4}$. Legionella spp es la causa de $2-15 \%$ de las neumonías en Europa y E.U.A., aunque se cree que puede estar sub-diagnosticada ${ }^{1}$.

La incidencia de neumonía por L. pneumophila es mayor en Europa que en América del Sur, donde el patógeno atípico más frecuente, tras Mycoplasma pneumoniae, es Chlamydia pneumoniae ${ }^{5,6}$. En cambio, se ha reportado L. pneumophila como la segunda causa de neumonías graves en una muestra estudiada en Santiago de Chile ${ }^{7}$. En España se ha descrito un incremento de la incidencia de neumonías adquiridas en la comunidad por Legionella spp; la mayoría de los casos ha ocurrido de forma aislada y sólo una fracción formó parte de brotes ${ }^{8}$.

El crecimiento óptimo de L. pneumophila con una temperatura ambiental de 35 a $37^{\circ} \mathrm{C}$ favorece que los casos de legionelosis aumenten a finales de verano y principios de otoño, y posiblemente sea la causa de que la enfermedad en España sea de mayor prevalencia en la zona mediterránea que en el área norte 9 .

Las manifestaciones clínicas de la legionelosis son variadas: fiebre alta, anorexia, malestar general, mialgias, síntomas digestivos (diarrea, vómitos, dolor abdominal), respiratorios (tos seca, disnea, expectoración) o neurológicos (disminución del estado de conciencia, delirio, síndrome confusional, cefalea). Es frecuente la insuficiencia renal y la afectación hepática, que suelen mejorar una vez superada la infección ${ }^{10}$.

La enfermedad afecta en especial a hombres de edad media, en particular a pacientes fumadores y/o con
Universidade da Coruña, 15006 A Coruña, España.

Complexo Hospitalario Universitario de A Coruña (CHUAC), SERGAS. Instituto de Investigación Biomédica de A Coruña (INIBIC). Servicio de Medicina Preventiva (MVRM, MFAR). Unidad de Epidemiología clínica y bioestadística (SPF, SPD, BLC, TSP).

Financiación: No hubo.

No existen conflictos de intereses de algún tipo en la realización de este trabajo.

Recibido: 4 de diciembre de 2014 Aceptado: 9 de mayo de 2015

Correspondencia a: María Virtudes Regueiro M. maria.virtudes.regueiro.mira@ sergas.es 
enfermedades crónicas. Se le concibe como una enfermedad oportunista, por esta preferencia por pacientes con co-morbilidad.

La tasa de incidencia en España se estima en torno a 7,8 casos por millón de habitantes, aunque se cree que estas cifras pueden estar sub-valoradas, debido a que puede manifestarse como un cuadro respiratorio leve que muchas veces no es investigado. Según la Red Nacional de Vigilancia Epidemiológica del Instituto de Salud Carlos III, la tasa de incidencia en el año 2009 fue de 2,89 por 100.000 habitantes ${ }^{11}$.

La legionelosis es la segunda causa de neumonía grave, que ingresa a la UCI, tras la neumonía neumocóccica, y la mortalidad de los pacientes ingresados en esta unidad ronda el $30 \%$, siendo en algún estudio hasta $60 \% \%^{2,4,11-22}$.

Con el objetivo de determinar las características de los pacientes diagnosticados de neumonía por L. pneumophila en el Complexo Hospitalario Universitario de A Coruña (CHUAC), y las variables asociadas a la necesidad de ingreso a la UCI y la mortalidad intra-hospitalaria se realizó este estudio, que contó con la autorización del Comité de Ética de Galicia (Código2014/197).

\section{Material y Métodos}

\section{Pacientes}

Se estudiaron de forma retrospectiva todos los pacientes diagnosticados como neumonía por L. pneumophila en el CHUAC desde enero de 2000 a diciembre de 2013, con ficha epidemiológica en el Servicio de Medicina Preventiva y Salud Pública del CHUAC. El diagnóstico había sido confirmado por determinación de antígeno de L. pneumophila en orina (inmunocromatografía de membrana Binax NOW para L. pneumophila serogrupo 1, con una sensibilidad aproximada de $97 \%$ en orina concentrada, y una especificidad de $97 \%)^{23}$ y hallazgos clínico-radiológicos compatibles con neumonía, según los criterios diagnósticos aprobados por la Sociedad Española de Neumología y Cirugía Torácica (SEPAR) ${ }^{8}$.

\section{Variables estudiadas}

Demográficas: edad, sexo, procedencia (Ciudad de A Coruña y Área Metropolitana, o fuera del área).

Co-morbilidad: Para el estudio de la co-morbilidad se utilizó el índice de co-morbilidad de Charlson que contiene 19 categorías de co-morbilidad ${ }^{24}$, estudiándose además el consumo excesivo de alcohol, consumo de tabaco actual, uso de corticosteroides sistémicos y antimicrobianos previos al diagnóstico. Se estimó además el filtrado glomerular (eGFR) calculado a través de la creatininemia utilizando la ecuación MDRD (Modification of Diet in Renal Disease), según la fórmula eGFR estimado $=175 \times$ (creatinina sérica)-1,154 $\times$ (edad)-0,203 x $(0,742$ si mujer) $\mathrm{x}(1,212 \text { si raza negra })^{25}$.

\section{Datos clínicos}

Fecha de inicio de los primeros síntomas, fecha de primera asistencia, servicio de ingreso, manifestaciones clínicas (fiebre, tos, disnea, cefalea, dolor costal, expectoración, dolor abdominal, diarrea, vómitos, confusión mental, malestar general), origen de la infección (comunitario o nosocomial cuando el ingreso fue al menos 10 días antes del inicio de los síntomas). Evolución: ingreso o no en UCI durante la hospitalización, necesidad de ventilación mecánica invasora (VMI) durante la estancia en UCI, y letalidad en el hospital.

\section{Hallazgos radiográficos: afectación uni o multilobular al ingreso}

Hallazgos analíticos: sodio plasmático, transaminasa glutámico-oxalacética (GOT), gammaglutamil transpeptidasa (GGT), fosfatasa alcalina sérica, uremia, creatininemia al ingreso, recuento de leucocitos, aclaramiento de creatinina (MDRD), lactato deshidrogenasa (LDH) sérica, creatinfosfoquinasa (CPK) sérica, presión parcial de oxígeno arterial $\left(\mathrm{PaO}_{2}\right)$, proteína $\mathrm{C}$ reactiva sérica (PCR).

Tratamiento antimicrobiano administrado en el diagnóstico y previo a la asistencia hospitalaria: $\beta$-lactámicos, macrólidos, aminoglucósidos, quinolonas y otros menos frecuentes.

\section{Análisis estadístico}

Se realizó un estudio descriptivo de las variables incluidas en el estudio, las variables cuantitativas se expresan como media $\pm \mathrm{DE}$, las variables cualitativas como valor absoluto y porcentaje con la estimación de su intervalo de confianza de $95 \%$. Se estudió la asociación entre variables cualitativas con el test de $\chi^{2}$ y en las variables cuantitativas, realizándose la comparación de medias, tras la comprobación de la normalidad con el test de Kolmogorov-Smirnov, con el test T de Student o U de Mann-Whitney, según procediera. Se realizó un análisis de regresión logística para determinar qué variables se asocian con los eventos de interés (mortalidad intrahospitalaria e ingreso a la unidad de cuidados intensivos). El análisis estadístico se realizó con los programas SPSS v19.0 y Epidat 3.1.

\section{Resulltados}

Durante el período de estudio se identificaron 240 casos con diagnóstico de neumonía por L. pneumophila, atendidos en el CHUAC desde enero de 2000 a diciembre de 2013. Sólo 4 (1,7\%) de estos casos estudiados presentaron un origen nosocomial, siendo el resto neumonías adquiridas en la comunidad. En todos los casos analizados, L. pneumophila fue el único microorganismo detectado como causante de la neumonía, no habiéndose efectuado un estudio sistemático de otras etiologías. 
La distribución estacional a lo largo de los años estudiados se muestra en la Figura 1, donde se objetivó un predominio de casos entre los meses de julio y noviembre.

Las características de la cohorte estudiada se describen en la Tabla 1. Existió un predominio de hombres sobre mujeres ( 88,3 vs $11,7 \%$ ), con una media de edad de 57,2 $\pm 15,4$ años, y una mediana de 54,5 años. La mediana del índice de Charlson fue de 2. Las manifestaciones clínicas más frecuentes fueron: fiebre $(92,5 \%)$, tos $(38,1 \%)$ y disnea $(33,9 \%)$. La mediana de la temperatura al ingreso era de $39^{\circ} \mathrm{C}$. La mayoría presentaba una afectación unilobular en la radiografía $(82,5 \%)$ y $50,3 \%$ tuvo valores de $\mathrm{PaO}_{2}$ menores de $60 \mathrm{mmHg}$ respirando aire ambiental. El 29,7\% presentaba una creatininemia menor de $60 \mathrm{~mL} / \mathrm{min} / 1,73$ $\mathrm{m}^{2}, \mathrm{y} 58,3 \%$ tuvo valores de sodio menores de $135 \mathrm{mEq} / \mathrm{L}$.

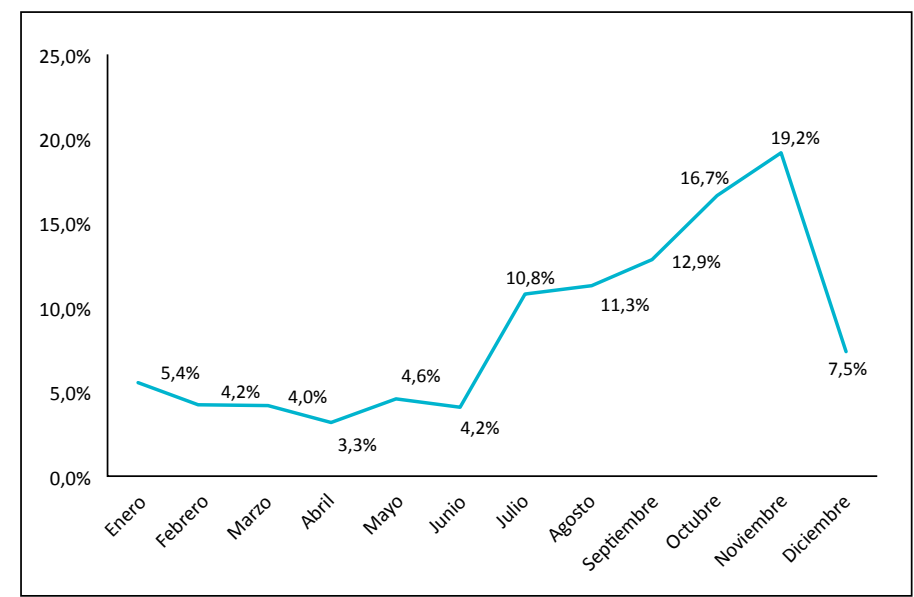

Figura 1. Distribución de los pacientes según mes de diagnóstico. Período 20002013.

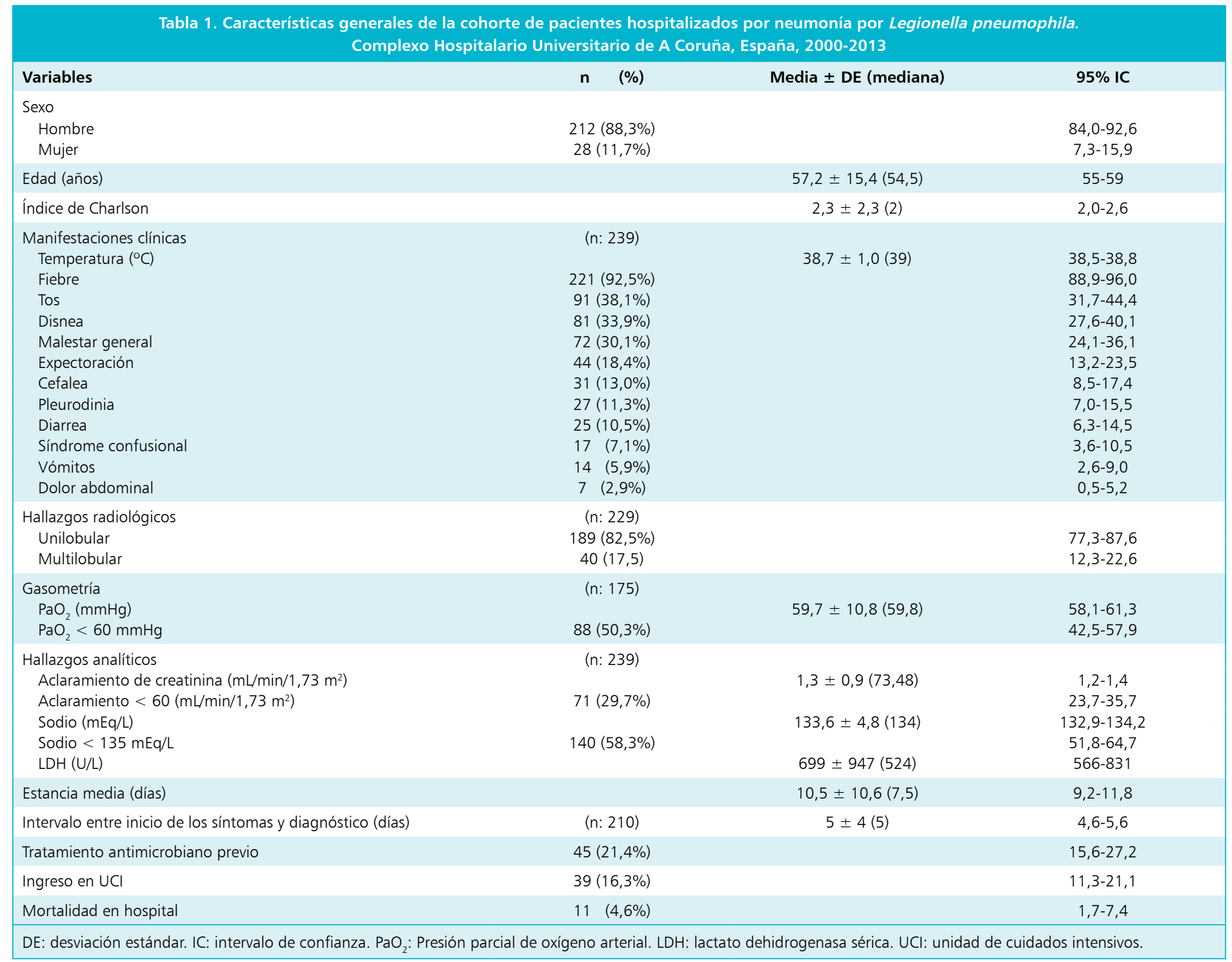


Tabla 2. Características generales de la cohorte de pacientes hospitalizados por neumonía por Legionella pneumophila según la necesidad o no de ingreso a la UCI durante el seguimiento. Complexo Hospitalario Universitario de A Coruña, España, 2000-2013

\begin{tabular}{|c|c|c|c|c|c|c|}
\hline Variables & $\begin{array}{l}\text { Ingreso en UCl } \\
\text { No }(n=201)\end{array}$ & $\begin{array}{l}\text { Ingreso en UCI } \\
\text { Sí }(n=39)\end{array}$ & OR crudo & $\begin{array}{l}\text { IC } 95 \% \\
\text { OR crudo }\end{array}$ & OR ajustado & $\begin{array}{c}\text { IC } 95 \% \\
\text { OR ajustado }\end{array}$ \\
\hline Sexo & n (\%) & n (\%) & & & & \\
\hline Hombre & $178(88,6 \%)$ & $34(87,2 \%)$ & 1 & - & - & - \\
\hline Mujer & $23(11,4 \%)$ & $5(12,8 \%)$ & 1,13 & 0,$40 ; 3,20$ & - & - \\
\hline Edad (años) & $\begin{array}{c}\text { Media } \pm \text { DE } \\
57,6 \pm 15,5\end{array}$ & $\begin{array}{c}\text { Media } \pm \text { DE } \\
54,7 \pm 14,4\end{array}$ & 0,98 & 0,$96 ; 1,01$ & 0,96 & 0,93; 0,99 \\
\hline Índice de Charlson & $2,2 \pm 2,3$ & $2,3 \pm 2,4$ & 0,98 & 0,$88 ; 1,34$ & - & - \\
\hline Temperatura $\left({ }^{\circ} \mathrm{C}\right)$ & $38,71 \pm 0,94$ & $38,57 \pm 1,30$ & 0,87 & 0,$62 ; 1,22$ & - & - \\
\hline Intervalo inicio síntomas y diagnóstico (días) & $5,17 \pm 3,5$ & $5,31 \pm 4,5$ & 1,01 & 0,$92 ; 1,10$ & - & - \\
\hline Estancia en hospital (días) & $8,35 \pm 6,8$ & $22,3 \pm 17,5$ & 1,11 & 1,$07 ; 1,16$ & - & - \\
\hline Cuadro clínico & n (\%) & n (\%) & & & - & - \\
\hline Fiebre & $189(94,0 \%)$ & $35(89,7 \%)$ & 0,55 & 0,$16 ; 1,82$ & - & - \\
\hline Tos & $76(37,8 \%)$ & $16(41,0 \%)$ & 1,14 & 0,$56 ; 2,30$ & - & - \\
\hline Disnea & $57(28,4 \%)$ & $25(64,1 \%)$ & 4,51 & 2,$19 ; 9,29$ & 4,33 & 1,$69 ; 11,03$ \\
\hline Cefalea & $27(13,4 \%)$ & $3(7,7 \%)$ & 0,53 & 0,$15 ; 1,86$ & - & - \\
\hline Pleurodinia & $24(11,9 \%)$ & $2(5,1 \%)$ & 0,39 & 0,$09 ; 1,76$ & - & - \\
\hline Expectoración & $38(18,9 \%)$ & $6(15,4 \%)$ & 0,78 & 0,$30 ; 1,99$ & - & - \\
\hline Diarrea & $21(10,4 \%)$ & $6(15,4 \%)$ & 1,55 & 0,$58 ; 4,15$ & - & - \\
\hline Confusión mental & $11(5,5 \%)$ & $6(15,4 \%)$ & 3,14 & 1,$08 ; 9,07$ & 5,86 & 1,$38 ; 24,74$ \\
\hline Malestar general & $64(31,8 \%)$ & $10(25,6 \%)$ & 0,73 & 0,$33 ; 1,60$ & - & - \\
\hline \multicolumn{7}{|l|}{ Hallazgos radiológicos } \\
\hline Unilobular & $163(85,8 \%)$ & $26(66,7 \%)$ & 1 & - & - & - \\
\hline Multilobular & $27(14,2 \%)$ & $13(33,3 \%)$ & 3,01 & 1,$38 ; 6,58$ & 2,64 & 1,$03 ; 6,75$ \\
\hline \multicolumn{7}{|l|}{ Factores de riesgo } \\
\hline Hepatopatía & $5(2,5 \%)$ & $4(10,3 \%)$ & 4,48 & 1,$14 ; 17,50$ & 7,13 & 1,$47 ; 34,61$ \\
\hline Gasometría & Media $\pm \mathrm{DE}$ & Media $\pm \mathrm{DE}$ & & & & \\
\hline $\mathrm{PaO}_{2}(\mathrm{mmHg})$ & $\begin{array}{c}60,2 \pm 9,6 \\
\text { n (\%) }\end{array}$ & $\begin{array}{c}57,8 \pm 14,8 \\
\text { n (\%) }\end{array}$ & 0,97 & 0,$94 ; 1,01$ & - & - \\
\hline $\mathrm{PaO}_{2}<60 \mathrm{mmHg}$ & $70 / 143(49,0 \%)$ & $18 / 32(56,3 \%)$ & 1,34 & 0,$62 ; 2,90$ & - & - \\
\hline Hallazgos analíticos & Media $\pm \mathrm{DE}$ & Media $\pm \mathrm{DE}$ & & & & \\
\hline LDH sérica (U/L) & $555,8 \pm 281,2$ & $1304,8 \pm 1996,9$ & 1,002 & 1,$001 ; 1,003$ & 1,002 & 1,$001 ; 1,003$ \\
\hline Aclaramiento creatinina $\left(\mathrm{mL} / \mathrm{min} / 1,73 \mathrm{~m}^{2}\right)$ & $77,0 \pm 30,6$ & $36,7 \pm 17,7$ & 0,98 & 0,$97 ; 0,99$ & 0,98 & 0,$97 ; 1,002$ \\
\hline Sodio $(\mathrm{mEq} / \mathrm{L})$ & $\begin{array}{c}133,5 \pm 4,2 \\
\text { n (\%) }\end{array}$ & $\begin{array}{c}134,2 \pm 7,1 \\
\text { n (\%) }\end{array}$ & 1,03 & 0,$96 ; 1,10$ & - & - \\
\hline Aclaramiento creatinina $<60\left(\mathrm{~mL} / \mathrm{min} / 1,73 \mathrm{~m}^{2}\right)$ & $48(24,0 \%)$ & $23(59,0 \%)$ & 4,55 & 2,$2 ; 9,3$ & - & - \\
\hline Sodio $<135 \mathrm{mEq} / \mathrm{L}$ & $121 / 201(60,2 \%)$ & $19(48,7 \%)$ & 0,62 & 0,$31 ; 1,25$ & - & - \\
\hline Antimicrobiano previo & $36 / 172(20,9 \%)$ & $9 / 38(23,7 \%)$ & 1,17 & 0,$51 ; 2,69$ & - & - \\
\hline
\end{tabular}

El 21,4\% recibió tratamiento antimicrobiano previo a acudir a urgencias, requiriendo $16,3 \%$ el ingreso en UCI durante la hospitalización.

Fallecieron 11 pacientes en el período del estudio, de lo que se infiere una tasa de letalidad hospitalaria de 4,6\%.

La utilización de antimicrobiano previo (Tablas 2 y 3) incrementó el riesgo de ingreso a UCI y disminuyó el riesgo de mortalidad, como se puede objetivar por los OR calculados, aunque sin alcanzar diferencias significativas. Tras el diagnóstico, $67,8 \%$ de los pacientes fue tratado con quinolonas llamadas "respiratorias".

En el análisis bivariado las variables que se asociaron significativamente con el ingreso a UCI (Tabla 2) fueron: la presencia de disnea, el síndrome confusional agudo, la afectación radiológica multilobular, el menor aclaramiento de creatinina y los valores de LDH sérica elevados. Los pacientes que ingresaron en UCI presentaron disnea $(64,1 \%)$, en comparación con $28,4 \%$ de los que no ingresaron. A su vez el síndrome confusional agudo fue más frecuente entre los que ingresaron que 
Tabla 3. Características generales de la cohorte de pacientes hospitalizados por neumonía por Legionella pneumophila según la presencia o no de muerte durante el seguimiento. Complexo Hospitalario Universitario de A Coruña, España, 2000-2013

\begin{tabular}{|c|c|c|c|c|c|c|}
\hline Variables & $\begin{array}{c}\text { Vivos } \\
(n=229)\end{array}$ & $\begin{array}{c}\text { Fallecidos } \\
(n=11)\end{array}$ & OR crudo & $\begin{array}{l}\text { IC } 95 \% \\
\text { OR crudo }\end{array}$ & OR ajustado & $\begin{array}{c}\text { IC } 95 \% \\
\text { OR ajustado }\end{array}$ \\
\hline Sexo & n (\%) & n (\%) & & & & \\
\hline Hombre & $202(88,2 \%)$ & $10(90,9 \%)$ & 1 & - & - & - \\
\hline Mujer & $27(11,8 \%)$ & $1(9,1 \%)$ & 0,7 & 0,$09 ; 6,0$ & - & - \\
\hline Edad (años) & $\begin{array}{l}\text { Media } \pm \text { DE } \\
56,79 \pm 15,3\end{array}$ & $\begin{array}{l}\text { Media } \pm \text { DE } \\
64,55 \pm 15,0\end{array}$ & 1,03 & 0,$9 ; 1,08$ & - & - \\
\hline Índice de Charlson & $2,2 \pm 2,3$ & $3,7 \pm 2,3$ & 1,28 & 0,$96 ; 1,71$ & 1,70 & 1,$13 ; 2,55$ \\
\hline Temperatura $\left({ }^{\circ} \mathrm{C}\right)$ & $38,7 \pm 1,0$ & $38,6 \pm 1,2$ & 0,92 & 0,$49 ; 1,70$ & - & - \\
\hline Intervalo inicio síntomas y diagnóstico (días) & $5,12 \pm 3,3$ & $6,6 \pm 8,0$ & 1,08 & 0,$96 ; 1,21$ & - & - \\
\hline Manifestaciones clínicas & n (\%) & n (\%) & & & & \\
\hline Fiebre & $216(94,3 \%)$ & $8(72,7 \%)$ & 0,16 & 0,$03 ; 0,67$ & - & - \\
\hline Tos & $89(38,9 \%)$ & $3(27,3 \%)$ & 0,59 & 0,$15 ; 2,28$ & - & - \\
\hline Disnea & $72(31,4 \%)$ & $10(90,9 \%)$ & 21,80 & 2,$73 ; 173,57$ & 13,36 & 0,$92 ; 192,38$ \\
\hline Cefalea & $28(12,2 \%)$ & $2(18,2 \%)$ & 1,59 & 0,$32 ; 7,76$ & - & - \\
\hline Expectoración & $42(18,3 \%)$ & $2(18,2 \%)$ & 0,98 & 0,$20 ; 4,74$ & - & - \\
\hline Diarrea & $24(10,5 \%)$ & $3(27,3 \%)$ & 3,20 & 0,$79 ; 12,89$ & - & - \\
\hline Vómitos & $13(5,7 \%)$ & $1(9,1 \%)$ & 1,66 & 0,$19 ; 13,98$ & - & - \\
\hline Confusión mental & $16(7,0 \%)$ & $1(9,1 \%)$ & 1,33 & 0,$16 ; 11,06$ & - & - \\
\hline Malestar general & $71(31,0 \%)$ & $8(72,7 \%)$ & 0,83 & 0,$21 ; 3,23$ & - & - \\
\hline \multicolumn{7}{|l|}{ Hallazgos radiológicos } \\
\hline Unilobular & $182(83,5 \%)$ & $7(63,6 \%)$ & 1 & - & - & - \\
\hline Multilobular & $36(16,5 \%)$ & $4(36,4 \%)$ & 2,88 & 0,$80 ; 10,38$ & - & - \\
\hline \multicolumn{7}{|l|}{ Gasometría } \\
\hline $\mathrm{PaO}_{2}<60 \mathrm{mmHg}$ & $84 / 167(50,3 \%)$ & $4 / 8(50,0 \%)$ & 0,98 & 0,$23 ; 4,08$ & - & - \\
\hline Da 0 ( & IVIeald I DE & Fieuld I De & & & & \\
\hline $\mathrm{PaO}_{2}(\mathrm{mmHg})$ & $59,8 \pm 10,5$ & $58,3 \pm 15,7$ & 0,98 & 0,$92 ; 1,05$ & - & - \\
\hline \multicolumn{7}{|l|}{ Hallazgos analíticos } \\
\hline LDH sérica (U/L) & $599,8 \pm 370,2$ & $2585,1 \pm 3562,6$ & 1,001 & 1,$001 ; 1,002$ & 1,002 & 1,$001 ; 1,003$ \\
\hline Aclaramiento creatinina $\left(\mathrm{mL} / \mathrm{min} / 1,73 \mathrm{~m}^{2}\right)$ & $77,0 \pm 30,6$ & $36,7 \pm 17,7$ & 0,94 & 0,$91 ; 0,97$ & - & - \\
\hline Sodio (mEq/L) & $\begin{array}{c}133,6 \pm 4,5 \\
\text { n (\%) }\end{array}$ & $\begin{array}{c}133,8 \pm 9,7 \\
\text { n (\%) }\end{array}$ & 1,008 & 0,$88 ; 1,14$ & - & - \\
\hline Aclaramiento creatinina $<60 \mathrm{~mL} / \mathrm{min} / 1,73 \mathrm{~m}^{2}$ & $61 / 227(26,9 \%)$ & $10(90,9 \%)$ & 27,37 & 3,$43 ; 218,36$ & - & - \\
\hline Sodio $<135 \mathrm{mEq} / \mathrm{L}$ & $136(59,4 \%)$ & $4(36,4 \%)$ & 0,39 & 0,$11 ; 1,37$ & - & - \\
\hline Antimicrobiano previo & $44 / 200(22,0 \%)$ & $1 / 10(10,0 \%)$ & 0,39 & 0,$049 ; 3,19$ & - & - \\
\hline Ingreso a la UCl & $30(13,1 \%)$ & $9(81,8 \%)$ & 29,85 & 6,$15 ; 144,85$ & - & - \\
\hline Ventilación mecánica invasora & $17(7,4 \%)$ & $9(81,8 \%)$ & 56,11 & 11,$21 ; 280,70$ & 31,44 & 3,$19 ; 309,61$ \\
\hline
\end{tabular}

los que no lo hicieron $(15,4$ vs $5,5 \%)$, así como la afectación radiológica multilobular (33,3 vs $14,2 \%)$. El aclaramiento de creatinina fue significativamente menor en los pacientes que ingresaron, en comparación con los que no lo hicieron $\left(36,7 \pm 17,7\left(\mathrm{~mL} / \mathrm{min} / 1,73 \mathrm{~m}^{2}\right)\right.$ vs 77,0 $\pm 30,6\left(\mathrm{~mL} / \mathrm{min} / 1,73 \mathrm{~m}^{2}\right)$. El 59,0\% de los que ingresaron a UCI tenía insuficiencia renal (GFR menor de $60 \mathrm{~mL} /$ $\min / 1,73 \mathrm{~m}^{2}$ ) en comparación con $24,0 \%$ de los que no lo hicieron. A su vez, los pacientes que ingresaron a UCI tuvieron valores de LDH sérica significativamente más elevados que los que no lo requirieron $(1.304,8 \pm 1.996,9$ $\mathrm{U} / \mathrm{L}$ vs $555,8 \pm 281,2 \mathrm{U} / \mathrm{L})$.

A pesar que el índice de Charlson en su conjunto no fue significativamente diferente entre los que ingresaron o no a la UCI, dentro de las enfermedades que tiene en consideración dicho score, la presencia de hepatopatía crónica tuvo mayor prevalencia en los pacientes que ingresaron a UCI, en comparación con los que no lo requirieron (10,3 vs 2,5\%). La hipoxemia también tuvo mayor prevalencia en los pacientes que ingresaron a UCI, 
Tabla 4. Factores pronósticos de ingreso a UCI o mortalidad en pacientes hospitalizados por neumonía por Legionella pneumophila. Complexo Hospitalario Universitario de A Coruña, España, 2000-2013

\begin{tabular}{|c|c|c|c|c|c|}
\hline Referencias & Año de publicación & Período de estudio & País & $\mathbf{n}$ & Factores pronósticos identificados \\
\hline \multicolumn{6}{|l|}{ Ingreso en UCI } \\
\hline Nuestro estudio & 2014 & $2000-2013$ & España & 240 & $\begin{array}{l}\text { Edad } \\
\text { Niveles elevados LDH sérica } \\
\text { Afectación radiográfica multilobular } \\
\text { Disnea } \\
\text { Síndrome confusional } \\
\text { Hepatopatía crónica }\end{array}$ \\
\hline Lettinga et $\mathrm{a}^{26}$ & 2002 & 1999 & Holanda & 141 & $\begin{array}{l}\text { Tabaquismo } \\
\text { Temperatura }>38,5^{\circ} \mathrm{C} \\
\text { Infiltrados bilaterales }\end{array}$ \\
\hline \multicolumn{6}{|l|}{ Mortalidad } \\
\hline Nuestro estudio & 2014 & $2000-2013$ & España & 240 & $\begin{array}{l}\text { Índice de Charlson } \\
\text { Intubación orotraqueal } \\
\text { Disnea } \\
\text { Niveles elevados LDH sérica }\end{array}$ \\
\hline Rello et $a^{36 *}$ & 2013 & $\begin{array}{l}2000-2002 \\
2008-2011\end{array}$ & España & 25 & Monoterapia antibiótica \\
\hline Viasus et $\mathrm{al}^{12}$ & 2013 & $1995-2010$ & España & 214 & $\begin{array}{l}\text { Fumador o ex-fumador } \\
\text { Antibioterapia inicial inapropiada } \\
\text { Tratamiento con macrólidos } \\
\text { Escala PSI alto riesgo IV-V }\end{array}$ \\
\hline Chidiac et $\mathrm{al}^{37}$ & 2012 & 2006-2007 & Francia & 540 & $\begin{array}{l}\text { Edad } \\
\text { Sexo femenino } \\
\text { Ingreso a UCI } \\
\text { Falla renal } \\
\text { Escala PSI alto riesgo IV-V } \\
\text { Corticoterapia } \\
\text { PCR sérica }>500 \mathrm{mg} / \mathrm{L}\end{array}$ \\
\hline Jespersen et $a^{2 * * *}$ & 2010 & $1995-2005$ & Dinamarca & 332 & $\begin{array}{l}\text { Índice de Charlson }>2 \\
\text { Edad }>65 \text { años } \\
\text { Auscultación pulmonar normal } \\
\text { Demora en el diagnóstico y tratamiento } \\
\text { Infección nosocomial }\end{array}$ \\
\hline Domínguez et $a^{38 * \star *}$ & 2009 & 1993-2004 & España & 1938 & $\begin{array}{l}\text { Edad }>70 \text { años } \\
\text { Cáncer }\end{array}$ \\
\hline Haeuptle et al ${ }^{18}$ & 2009 & $2002-2007$ & Suiza & 29 & Niveles procalcitonina $>1,5 \mathrm{ng} / \mathrm{mL}$ \\
\hline Gudiol et al ${ }^{16 * *}$ & 2009 & $1985-2007$ & España & 14 & Demora en el diagnóstico y tratamiento \\
\hline Benito et $\mathrm{al}^{13}$ & 2003 & $1997-2001$ & España & 97 & $\begin{array}{l}\text { Frecuencia respiratoria }>30 \mathrm{rpm} \\
\text { Urea }>60 \mathrm{mg} / \mathrm{dL} \\
\mathrm{PaO}_{2}<60 \mathrm{mmHg}\end{array}$ \\
\hline Howden et al ${ }^{20}$ & 2003 & 2000 & Australia & 104 & $\begin{array}{l}\text { Falla cardíaca previo al ingreso } \\
\text { Enfermedad renal previa }\end{array}$ \\
\hline Gacouin et al ${ }^{19 *}$ & 2002 & $1990-2000$ & Francia & 43 & $\begin{array}{l}\text { SAPS } \|>46 \\
\text { Duración de síntomas antes de UCI }>5 \text { días }\end{array}$ \\
\hline Tkatch et al ${ }^{39}$ & 1998 & 1986-1994 & EE.UU. & 40 & $\begin{array}{l}\text { Origen nosocomial } \\
\text { Intubación orotraqueal } \\
\text { Absceso o cavitación pulmonar } \\
\text { Efusión pleural }\end{array}$ \\
\hline El-Ebiary et al ${ }^{17 *}$ & 1997 & 1983-1991 & España & 84 & $\begin{array}{l}\text { Hiponatremia }<136 \mathrm{mg} / \mathrm{dL} \\
\text { APACHE II }>15\end{array}$ \\
\hline
\end{tabular}




\begin{tabular}{|c|c|c|c|c|c|c|}
\hline Referencias & Año de publicación & Años de estudio & País & $\begin{array}{c}\text { Mortalidad UCI } \\
\%\end{array}$ & $\begin{array}{c}\text { Mortalidad general } \\
\%\end{array}$ & $n$ \\
\hline Muestra estudiada & 2014 & $2000-2013$ & España & 23,1 & 4,6 & 240 \\
\hline Viasus et al'12 & 2013 & $1995-2010$ & España & 17,8 & 6,1 & 214 \\
\hline Chidiac et al ${ }^{137}$ & 2012 & $2006-2007$ & Francia & & 8,1 & 540 \\
\hline Jespersen et $\mathrm{al}^{2}$ & 2010 & $1995-2005$ & Dinamarca & 42,6 vs $61,7^{*}$ & 12,9 vs $33,3^{*}$ & 272 \\
\hline Gudiol et al ${ }^{16}$ & 2009 & $1985-2007$ & España & & 14,3 & 14 \\
\hline Haeuptle et al ${ }^{18}$ & 2009 & $2002-2007$ & Suiza & 55 & 14 & 29 \\
\hline Sopena et $\mathrm{al}^{22}$ & 2007 & $1994-2004$ & España & 16,1 vs $4,4^{\text {** }}$ & 5,9 vs $1,8^{* *}$ & 251 \\
\hline Sopena et $a^{15}$ & 2007 & $1994-2004$ & España & & 11,2 vs $4,8^{* * *}$ & 158 \\
\hline Falcó et al ${ }^{34}$ & 2006 & $2001-2004$ & España & 14 & 5,3 & 116 \\
\hline Benito et $\mathrm{al}^{13}$ & 2003 & $1997-2001$ & España & 37,1 & 12,5 & 97 \\
\hline García-Fulgueiras et al ${ }^{4}$ & 2003 & 2001 & España & & 1 & 449 \\
\hline Howden et $\mathrm{al}^{20}$ & 2003 & 2000 & Australia & & 5,8 & 104 \\
\hline Gacouin et al ${ }^{19}$ & 2002 & $1990-2001$ & Francia & 33 & & 43 \\
\hline Lettinga et $\mathrm{al}^{26}$ & 2002 & 1999 & Holanda & 36 & 13 & 141 \\
\hline Formica et $a^{21}$ & 2001 & 1995-1999 & Australia & & 12 & 212 \\
\hline El-Ebiary et al ${ }^{17}$ & 1997 & 1983-1991 & España & 31 vs $27^{*}$ & & 84 \\
\hline Pedro-Botet et al'14 & 1995 & 1983-1992 & España & & 6 vs $18^{*}$ & 158 \\
\hline Falcó et al ${ }^{33}$ & 1991 & 1988-1989 & España & & 10 & 30 \\
\hline
\end{tabular}

sin alcanzar diferencia estadísticamente significativa (56,3 vs 49,0\%).

Tras ajustar un modelo multivariado de regresión logística, para predecir las variables asociadas al ingreso a UCI, las variables que tuvieron un efecto independiente para predecir el ingreso en UCI fueron: la menor edad (OR: 0,96), hepatopatía crónica (OR: 7,13), presencia de disnea (OR: 4,33), el síndrome confusional agudo (OR: 5,86), afectación radiológica multilobular (OR: $2,64)$ y los valores de LDH sérica elevados (OR: 1,002) (Tabla 2).

Como previamente hemos comentado, la letalidad en el hospital resultó de 4,6\% (95\% IC 1,7-7,4). Las variables de ingreso significativamente asociadas a mortalidad en el análisis bivariado (Tabla 3 ) fueron la co-morbilidad de Charlson, la presencia de fiebre, disnea, el aclaramiento de creatinina bajo, LDH sérica elevada, ingreso a la UCI y ventilación mecánica invasora. Los pacientes que fallecieron presentaban un score de Charlson más elevado que los que no lo hicieron (3,7 vs 2,2). Aunque no hubo diferencia estadísticamente significativa en la temperatura al ingreso, la presencia auto-declarada de fiebre fue menos frecuente en los pacientes fallecidos ( 72,7 vs $94,3 \%$ ). Por otra parte, la presencia de disnea fue claramente mayor en los que fallecieron que en los que no ( 90,9 vs $31,0 \%)$. A su vez el aclaramiento de creatinina fue menor en los que fallecieron que en los que no $(36,7 \pm 17,7 \mathrm{~mL} / \mathrm{min} / 1,73$ $\mathrm{m}^{2}$ vs 77,0 $\pm 30,6 \mathrm{~mL} / \mathrm{min} / 1,73 \mathrm{~m}^{2}$ ) y la $\mathrm{LDH}$ sérica fue más elevada. De los pacientes que fallecidos, $81,8 \%$ había ingresado a la UCI, en comparación con los no fallecidos, de los que sólo ingresó $13,1 \%$. La misma tendencia se objetiva con la ventilación mecánica invasora. A pesar de no existir diferencias significativas, los pacientes fallecidos eran mayores (64,5 $\pm 15,0$ años vs 56,7 $\pm 15,3$ años).

Tras ajustar un modelo multivariado de regresión logística, las variables que mantuvieron un efecto independiente para predecir la mortalidad fueron: el índice de co-morbilidad de Charlson (OR: 1,70), la necesidad de ventilación mecánica invasora $(\mathrm{OR}: 31,44)$ y los valores elevados de LDH sérica (OR: 1,002) (Tabla 3).

\section{Discusión}

La incidencia de la neumonía por L. pneumophila en España se mantiene estable en los últimos años, tras un 
aumento inicial a partir de 1997, atribuible al uso más frecuente de la detección en orina de antígeno específico de L. pneumophila serogrupo 1, que ha favorecido el diagnóstico de las infecciones por esta bacteria. En nuestro estudio se observa la misma tendencia, y hay mayor número de casos en verano y principios de otoño, similar a lo descrito en otros estudios ${ }^{12}$ y en la literatura médica. A lo largo del período estudiado por nosotros no se detectaron brotes.

Las manifestaciones clínicas más frecuentes en este estudio fueron: fiebre elevada, tos seca y disnea, hallazgos consistentes con las publicaciones revisadas ${ }^{13}$. El 21,4\% de los pacientes había recibido tratamiento antimicrobiano previo al ingreso, una cifra mayor que la reflejada en otros estudios $^{13}$. Esta exposición previa a antimicrobianos no la hemos podido asociar con la necesidad de ingreso en UCI o mortalidad aunque otros autores sí la relacionan ${ }^{12}$

En nuestra serie, 16,3\% de los pacientes necesitó ingreso en UCI a lo largo de su estancia hospitalaria, una cifra similar a otros reportes ${ }^{12,31}$ Algunos autores ${ }^{19}$ encontraron que la duración de los síntomas antes del ingreso se asocia a necesidad de ingreso en UCI. En nuestra serie, la demora entre el inicio de los síntomas y la solicitud de asistencia en nuestro hospital no se asoció con el ingreso en UCI ni con la mortalidad.

Hemos encontrado pocos trabajos que estudien factores predictores del ingreso a la UCI en esta patología. En nuestra serie, la edad avanzada se asoció a menor probabilidad de ingreso a la UCI. Esto puede explicarse por la mayor co-morbilidad de los pacientes mayores, que con frecuencia no los hace subsidiarios de atención en unidades de pacientes críticos. A su vez, la presencia de disnea y alteración del nivel de conciencia en la admisión al hospital se asociaron en forma independiente para predecir el ingreso a la UCI. Estos pacientes necesitan con mayor frecuencia ventilación mecánica invasora. En otros estudios se han encontrado resultados equiparables ${ }^{26}$, como se expresa en la Tabla 4.

En diferentes publicaciones realizadas en Chile se pone igualmente de manifiesto cómo en neumonías adquiridas en la comunidad, la edad y la co-morbilidad incrementan la probabilidad de ingreso en UCI y de mortalidad $^{27,28}$.

En cuanto a las co-morbilidades, no se encontró relación con el índice de co-morbilidad de Charlson y la posibilidad de ingreso en UCI, pero la existencia de hepatopatía crónica sí incrementó dicha posibilidad de ingreso.

Las concentraciones plasmáticas elevadas de LDH resultaron ser un factor pronóstico, tanto de ingreso en UCI como de mortalidad. Esto se podría interpretar como marcador de inflamación y destrucción tisular, por lo que estaría más elevado en pacientes graves o quienes fallecerán, quienes experimentan una mayor afectación sistémica por la infección.
Aunque se han descrito concentraciones plasmáticas de LDH más elevadas en formas más graves de legionelosis ${ }^{29,30}$ y también como factor predictor de neumonía por L. pneumophila ${ }^{31}$, no hemos encontrado estudios que hallasen una relación significativa de la LDH sérica con un peor pronóstico. No obstante, hay estudios en pacientes con neumonía por virus influenza H1N1 en los que las concentraciones plasmáticas elevadas de LDH sérica sí se han relacionado con la mortalidad en estos pacientes ${ }^{32}$.

En nuestra casuística, la tasa de letalidad fue $4,6 \%$, cifra similar a la de la mayoría de los estudios revisados, aunque es mayor en pacientes ingresados en UCI $(23,1 \%)$. La mortalidad que hemos encontrado en otras publicaciones varía entre 1 y $33,3 \%$, y la mortalidad en UCI entre 4 y $61,7 \%^{2,12-22,33-37}$, datos recogidos en la Tabla 5. Estas diferencias se deben a que en algunos de estos estudios, los pacientes reunían condiciones particulares, tales como ser receptores de trasplante de órgano sólido, o se comparaban por franjas de edad o por casos adquiridos en la comunidad o nosocomiales.

En cuanto al índice de co-morbilidad de Charlson, hemos encontrado que es una variable independiente y predictora de mortalidad en el hospital, al igual que en otros estudios ${ }^{2}$. Una neumonía por Legionella en el contexto de un paciente con mucha co-morbilidad va a tener mayor mortalidad, debido a las enfermedades acompañantes. En nuestros casos, el aclaramiento de creatinina es una variable predictora de mortalidad en el análisis univariado, aunque no en el multivariado. Sin embargo, la disfunción renal aguda es más frecuente en los pacientes que fallecen o ingresan a la UCI, lo que hace pensar en una afectación renal mayor por la infección. La LDH sérica se ha descrito en algún estudio como buen marcador de daño renal agudo ${ }^{38}$.

Entre las limitaciones del estudio se deben señalar las propias de las series retrospectivas con sesgos de información realizadas en un solo centro. La concomitancia con otros microorganismos no fue analizada, lo que podría generar también un sesgo de información, aunque la consistencia de los resultados del estudio con otras publicaciones no parece afectar la validez externa. Por otra parte, la utilización de antimicrobianos previos con los eventos de interés (ingreso en UCI o mortalidad) no llegó a alcanzar significación estadística por el reducido número de eventos.

Este estudio pone de manifiesto la estacionalidad de la infección, la frecuencia de fiebre elevada incluso en ausencia de síntomas respiratorios, así como la probabilidad de ingreso en UCI aumentada en pacientes jóvenes, con hepatopatía crónica, disnea o confusión y, finalmente, describimos que la mortalidad se asocia a la co-morbilidad, la necesidad de ventilación mecánica y cifras elevadas de LDH sérica. 


\section{Resumen}

Introducción: La legionelosis es una enfermedad bacteriana multisistémica, causante de neumonías con mortalidad elevada en pacientes con comorbilidad e ingresos en Uunidad de Cuidados Intensivos (UCI). Objetivo: Determinar factores pronósticos de mortalidad o ingreso en UCI. Material y Métodos: Estudio de seguimiento retrospectivo de pacientes diagnosticados de neumonía por Legionella pneumophila en Complexo Hospitalario Universitario de A Coruña (España). Período 2000-2013 ( $\mathrm{n}=240)$, con análisis de regresión logística multivariada. Resultados: La edad media fue 57,2 \pm 15,4 años, 88,3\% fueron hombres. La puntuación media de comorbilidad (score Charlson) fue 2,3 $\pm 2,3$. Existe clara estacionalidad. La clínica predominante fue fiebre
$(92,5 \%)$, tos seca $(38,1 \%)$ y disnea $(33,9 \%)$. El $29,7 \%$ presentó aclaramiento de creatinina $<60 \mathrm{~mL} / \mathrm{min} / 1,73$ $\mathrm{m}^{2}$ y el 58,3\% sodio $<135 \mathrm{mEq} / 1$. Un 16,3\% ingresó en UCI, precisando ventilación mecánica invasiva el 10,8\%. La mortalidad global fue 4,6\% y de $23,1 \%$ en ingresados en UCI. Variables asociadas para predecir ingreso en UCI fueron menor edad $(\mathrm{OR}=0,96)$, hepatopatía $(\mathrm{OR}=7,13)$, disnea $(\mathrm{OR}=4,33)$, síndrome confusional $(\mathrm{OR}=5,86)$ y lactato deshidrogenasa elevada $(\mathrm{OR}=1,002)$. Las variables asociadas a mortalidad intrahospitalaria fueron índice de Charlson $(\mathrm{OR}=1,70)$, ventilación mecánica invasiva $(\mathrm{OR}=31,44)$ y cifras elevadas de lactato deshidrogenasa $(\mathrm{OR}=1,002)$. Discusión: Pacientes jóvenes, con hepatopatía, disnea o confusión tienen más probabilidad de ingresar en UCI. Comorbilidad, ventilación mecánica y lactato deshidrogenasa elevada se asocian a mortalidad.

\section{Referencias bibliográficas}

1.- Beauté J, Zucs P, de Jong B. Legionnaires disease in Europe, 2009-2010. European Legionnaires `Disease Surveillance Network 2013; 18 (20417).

2.- Jespersen $\mathrm{S}$, Sogaard O, Schonheyder H, Fine M, Ostergaad L. Clinical features and predictors of mortality in admitted patients with community and hospital-acquired legionellosis: A Danish historical cohort study. BMC Infect Dis 2010; 10: 124.

3.- Ulloa M. Legionella pneumophila. Rev Chilena Infectol 2008; 25 (5): 208.

4.- García-Fulgueiras A, Navarro C, Fenoll D, García J, González-Diego P, Jiménez-Buñuales $\mathrm{T}$, et al. Legionnaires' disease outbreak in Murcia, Spain. Emerg Infect Dis 2003; 9 (8): 915-21.

5.- Díaz A, Barría P, Niederman M, Restrepo M, Dreyse J, Fuentes G, et al. Etiology of community-acquired pneumonia in hospitalized patients in Chile. The increasing prevalence of respiratory viruses among classic pathogens. CHEST 2007; 131: 779-87.

6.- Arnold F, Summersgill J, La Joie A, Peyrani P, Marrie T, Rossi P, et al. A worldwide perspective of atypical pathogens in community-acquired pneumonia. Am J Respir Crit Care Med 2007; 175: 1086-93.

7.- Arancibia F, Cortés C, Valdés M, Cerda J, Hernández A, Soto L, et al. Importance of Legionella pneumophila in the etiology of severe community-acquired pneumonia in Santiago, Chile. CHEST 2014; 145: 290-6.

8.- Alfageme I, Aspa J, Bello S, Blanquer J, Blanquer R, Borderías L, et al. Normativa para el diagnóstico y el tratamiento de la neumonía adquirida en la comunidad. Sociedad Española de Neumología y Cirugía Torácica
(SEPAR). Arch Bronconeumol 2005; 41 (5): 272-89.

9.- Torres A, Caylà J A. Diagnostic testing and careful investigation of cases are needed to control unsuspected Legionnaires' disease outbreaks. Arch Bronconeumol 2002; 38 (1): 1-3.

10.- Daumas A, El-Mekaoui F, Bataille S, Caporossi L. Acute tubulointersticial nephritis complicating Legionnaires disease: a case report. J Med Case Rep [Internet] 2012; 6 (1): [100 p.].

11.- Red Nacional de Vigilancia Epidemiológica. Centro Nacional de Epidemiología. Legionelosis. Situación general y evolución de la enfermedad en España. Datos de la Red Nacional de Vigilancia Epidemiológica. Datos actualizados en noviembre de 2010. Instituto de Salud Carlos III; 2010.

12.- Viasus D, Di Yacovo S, García-Vidal C, Verdaguer R, Manresa F, Dorca J, et al. Community-acquired Legionella pneumophila pneumonia. A single-center experience with 214 hospitalized sporadic cases over 15 years. Medicine 2013; 92 (1): 51-60.

13.- Benito J, Montejo J, Cancelo L, Zalacaín R, López L, Fernández Gil de Pareja J, et al. Neumonía comunitaria por Legionella pneumophila serogrupo 1: 97 casos 2003; 21 (8): 394-400.

14.- Pedro-Botet M, Sabria-Leal M, Haro M, Rubio C, Giménez G, Sopena N, et al. Nosocomial and community-acquired Legionella pneumonia: clinical comparative analysis. Eur Respir J 1995; 8: 1929-33.

15.- Sopena N, Force L, Pedro-Botet M, Barrufet P, Sauca G, García-Núñez M, et al. Sporadic and epidemic community legionellosis: two faces of the same illness. Eur Respir J 2007; 29: 138-42.
16.- Gudiol C, García-Vidal C, Fernández-Sabé N, Verdaguer R, Lladó L, Roca J, et al. Clinical features and outcomes of Legionnaires disease in solid organ transplant recipients. Transpl Infect Dis 2009; 11: 78-82.

17.- El-Ebiary M, Sarmiento X, Torres A, Nogué S, Mesalles E, Bodí M, et al. Prognostic factors of severe Legionella pneumonia requiring admission to ICU. Am J Respir Crit Med 1997; 156: 1467-72.

18.- Haeuptle J, Zaborsky R, Fiumefreddo R, Trampuz A, Steffen I, Frei R, et al. Prognostic value of procalcitonin in Legionella pneumonia. Eur J Clin Microbiol Infect Dis 2009; 28: 55-60.

19.- Gacouin A, Le Tulzo Y, Lavoue S, Camus C, Hoff J, Bassen R, et al. Severe pneumonia due to Legionella pneumophila: prognostic factors, impact of delayed appropriate antimicrobial therapy. Intensive Care Med 2002; 28: 686-91.

20.- Howden B, Stuart R, Tallis G, Bailey M, Johnson P. Treatment and outcome of 104 hospitalized patients with legionnaires disease. Intern Med J 2003; 33: 484-8.

21.- Formica N, Yates M, Beers M, Carnie J, Hogg G, Ryan N, et al. The impact of diagnosis by legionella urinary antigen test on the epidemiology and outcomes of Legionnaires'disease. Epidemiol Infect 2001; 127: 275-80.

22.- Sopena N, Pedro-Botet L, Mateu L, Tolschinsky G, Rey-Joly C, Sabriá M. Community-acquired Legionella pneumonia in elderly patients: characteristics and outcome. J Am Geriatr Soc 2007; 55: 114-9.

23.- Molinos L. Detección de antígenos en la orina. Arch Bronconeumol 2006; 42 (3): 101-3.

24.- Charlson M, Pompei P, Ales K, MacKenzie C. 
A new method of classifying prognostic comorbidity in longitudinal studies: development and validation. J Chronic Dis 1987; 40 (5): 373-83.

25.- Levey A, Coresh J, Greene T, Stevens L, Zhang Y, Hendriksen S, et al. Using standarized serum creatinine values in the modification of diet in renal disease study equation for estimating glomerular filtration rate. Ann Intern Med 2006; 145 (4): 24-54.

26.- Lettinga K, Verbon A, Weverling G, Schellekens J, Den Boer J, Yzerman E. Legionnaires disease at a Dutch flower show: prognostic factors and impact of therapy. Emerg Infect Dis 2002; 8 (12): 1448-54.

27.- Díaz A, Álvarez M, Callejas C, Rosso R, Schnettler K, Saldías F. Clinical picture and prognostic factors for severe communityacquired pneumonia in adults admitted to the intensive care unit. Arch Bronconeumol 2005; 41 (1): 20-6.

28.- Saldías F, Mardóñez J, Marchesse M, Viviani P, Farías G, Díaz A, et al. Neumonía adquirida en la comunidad en pacientes adultos hospitalizados. Presentación clínica y factores pronósticos. Rev Med Chile 2002; 130 (12): 1373-82.

29.- Van Arsdall J, Wunderlich H, Melo J,
Nagar D, Ferris F, Raff M. The protean manifestations of Legionnaires 'disease. J Infect 1983; 7 (1): 51-62.

30.- Agulló-Ortuño M, García-Mancebo M, Montes-Ares O, Noguera-Velasco J. Biochemical and immunologic features of an outbreak of Legionnaires disease: comparative study between community-acquired pneumonias. Diagn Microbiol Infect Dis 2006; 56: 7-11.

31.- Fiumefreddo R, Zaborsky R, Haeuptle J, Christ-Crain M, Trampuz A, Steffen I, et al. Clinical predictors for Legionella in patients presenting with community-acquired pneumonia to the emergency department. BMC Pulm Med 2009; 9 (4). doi: 10.1186/1471-2466-9-4.

32.- Ríos F, Estenssoro E, Villarejo F, Valentini R, Aguilar L, Pezzola D, et al. Lung function and organ dysfunctions in 178 patients requiring mechanical ventilation during the 2009 influenza A (H1N1) pandemic. Critical Care 2011; 15 (4): R201. doi: 10.1186/cc10369.

33.- Falcó V. Legionella pneumophila. A cause of severe community-acquired pneumonia. CHEST 1991; 100 (4): 1007-11.

34.- Falcó V, Molina I, Juste C, Crespo M, Almirante B, Pigrau C, et al. Tratamiento de la neumonía por Legionella pneumophila.
¿Macrólidos o quinolonas? Enferm Infecc Microbiol Clin 2006; 24 (6): 360-4.

35.- Zager R, Johnson A, Becker K. Renal cortical lactate dehydrogenase: a useful, accurate, quantitative marker of in vivo tubular injury and acute renal failure 2013; PLoS One 2013; 8 (6): e66776.

36.- Rello J, Gattarelo S, Souto J, Sole-Violan J, Valles J, Peredo R. Community-acquired Legionella pneumonia in the intensive care unit: impact on survival of combined antibiotic therapy. Med Intensiva 2013; 37 (5): $320-6$

37.- Chidiac $\mathrm{C}, \mathrm{Che} \mathrm{D}$, Pires-Cronenburger $\mathrm{S}$, Jarraud S, Campese C, Bissery A, et al. Factors associated with hospital mortality in community-acquired legionellosis in France. Eur Respir J 2012; 39: 963-70.

38.- Domínguez A, Álvarez J, Sabria M, Carmona G, Torner N, Oviedo M. Factors influencing the case-fatality rate of Legionnaires disease. Int J Tuberc Lung Dis 2009; 13 (3): 407-12.

39.- Tkatch L, Kusne S, Irish W, Krystofiak S, Wing E. Epidemiology of Legionella pneumonia and factors associated with Legionella-related mortality at a Tertiary Care Center. Clin Infect Dis 1998; 27: 1479-86. 